\title{
Prevalence of Oral Complications occurring in a Population of Pediatric Cancer Patients receiving Chemotherapy
}

\author{
${ }^{1}$ Kapil Gandhi, ${ }^{2}$ Geetika Datta, ${ }^{3}$ Shilpa Ahuja, ${ }^{4}$ Tanvi Saxena, ${ }^{5}$ Ankush G Datta
}

\begin{abstract}
Multiagent chemotherapy, radiotherapy, or a combination of both are the contemporary methods of cancer treatment. With medical advancements, though cure rates have increased considerably, focus is now shifted to the potential early and late complications of the same. The aim of this study was to assess the early oral complications in pediatric patients receiving chemotherapy. Sixty-two children with cancer undergoing chemotherapy with the mean age of $7.42 \pm 3.6$ years were included in the study. The various types of malignancies and oral problems during chemotherapy were recorded in the subjects. The most commonly encountered malignancy was acute lymphoblastic leukemia at $35.5 \%$. Various oral and associated complications like mucosal inflammation with ulcerations, oral pain, xerostomia, and secondary infections were commonly seen, with mucositis being the most commonly observed complication in $58.1 \%$ of the subjects undergoing chemotherapy. Clinical importance of timely medical and dental interventions by a multidisciplinary team involving a pediatric dentist at different stages of anticancer treatment is also emphasized to minimize discomfort, increase treatment compliance, and improve the quality of life of pediatric patients.
\end{abstract}

Keywords: Chemotherapy, Malignancy, Oral complications, Pediatric.

How to cite this article: Gandhi K, Datta G, Ahuja S, Saxena T, Datta AG. Prevalence of Oral Complications occurring in a Population of Pediatric Cancer Patients receiving Chemotherapy. Int Int J Clin Pediatr Dent 2017;10(2):166-171.

Source of support: Nil

Conflict of interest: None

\section{INTRODUCTION}

Thriving on more than 10 million patients worldwide, cancer has definitely surfaced as a major public health

\footnotetext{
${ }^{1}$ Professor, ${ }^{2}$ Reader, ${ }^{3,4}$ Senior Lecturer, ${ }^{5}$ Ex-Senior Lecturer

${ }^{1-4}$ Department of Pedodontics and Preventive Dentistry Inderprastha Dental College \& Hospital, Ghaziabad, Uttar Pradesh, India

${ }^{5}$ Department of Orthodontics and Dentofacial Orthopedics Inderprastha Dental College \& Hospital, Ghaziabad, Uttar Pradesh, India

Corresponding Author: Kapil Gandhi, Professor, Department of Pedodontics and Preventive Dentistry, Inderprastha Dental College \& Hospital, Ghaziabad, Uttar Pradesh, India, Phone: +9126145137, e-mail: drkapilgandhi@gmail.com
}

issue. ${ }^{1}$ In modern times, cancer has been widely diagnosed in children even though it is rarely considered as a childhood disease. Cancer is increasingly becoming a cause of death of children aged between 1 and 14 years; also the incidence of cancer is amounting to an average increase of $1 \%$ every year. ${ }^{2}$ The research shows that 1.6 to $4.8 \%$ of all cancers in India is diagnosed in children aged below 15 years; however, the overall incidence of 38 to 124 per million children per year is lower than that in the developed world. ${ }^{3}$ Despite the technological advancements in diagnosis and treatment of this disease, the plight of cancer does not seem to cease in the near future.

The developing countries are plagued by peculiar problems like population increase, lack of education, poverty, poor oral hygiene, and multiple health problem that contribute to higher occurrences of cancer. Eightyfive percent of pediatric cancer cases are concentrated in developing countries. ${ }^{4}$ Studies show that instances of cancer are more common in male child than in female child, with the male to female ratio being around 1.2:1 in most of the resource-rich countries. ${ }^{5}$ In India, the most common cancer affecting children is leukemia, with the relative proportion varying from 25 to $40 \%$. Out of the reported leukemias, 60 to $85 \%$ are cases of acute lymphoblastic leukemia (ALL). ${ }^{3}$

With accomplished advances in the treatment of childhood cancer, there is an increase in young population who are successfully cured of their disease with the aid of various chemotherapeutic agents. With time, focus has shifted to mitigation of early and long-term side effects of several available treatment modalities. ${ }^{6}$

Ideally, a chemotherapeutic agent must completely erase all malignant cells. However, unavailability of anticancer drugs with such sparing effects has resulted in some damage to normal tissue. This can be particularly found in those tissues where rapid cell division normally occurs, such as oral and intestinal mucosa, bone marrow, hair follicle, liver, and testis. ${ }^{7}$

An array of significant therapy-related factors, viz., the type of chemotherapeutic agents used for therapy, the total dosage of the drug used, the frequency of drug administration, and other treatment modalities, such as radiotherapy used concomitantly with chemotherapy, 
affect the development of stomatotoxicity. ${ }^{8,9}$ Oral cavity is frequently affected with complications from cancer, or secondary cancer treatment. There has been substantial variety in the literature of reported incidences of such complications; however, it has been noted that children are most commonly affected by these complications. ${ }^{10}$ The factors affecting the severity of these complications include age of child, type of malignancy, condition of oral cavity before treatment, and the level of oral care during anticancer therapy. ${ }^{11,12}$

The common oral side effects include oral mucositis, intraoral infections, dry mouth, salivary gland inflammation, intraoral hemorrhage, and mucosal bleeding. ${ }^{13}$ Considering these factors and lack of existing information, a study was designed to investigate the prevalence and nature of orodental problems occurring in a population of pediatric cancer patients receiving chemotherapy in the city of Indore.

\section{MATERIALS AND METHODS}

Sixty-two children between the age of 1 and 14 years who are receiving chemotherapy for malignancy and had complete treatment records in different hospitals treating pediatric cancer patients across Indore in Madhya Pradesh state were randomly selected and included in the study. Exclusion criteria included the children who were suffering from oral cancer and also children who were uncooperative. Data were collected on the basis of age, gender, malignancy type (solid tumor or hematologic malignancy), and chemotherapeutic drug administered (type, dose, and number of chemotherapeutic cycles) from hospital records.

Current blood data with particular attention to the white blood cell count, the platelet count (PC), and the hemoglobin level were recorded. Patient complaints, such as dry mouth and difficulty in swallowing, mastication, or in speech and pain were recorded.

Informed consent was secured from the parents and the treating oncologist prior to the examination of the patients. Patients were examined using sterile equipment and material, under day light or torch light as they are lying or sitting on the bed. The examination was conducted by a team of two examiners, which consisted of a trained doctor and an assistant. The examination was done using latex gloves, plain mouth mirror, probes, periodontal probes, tweezers, containers, cotton/gauze to remove any food debris and to dry the mucosa.

Scoring of chemotherapy-induced oral mucositis was done according to the World Health Organization (WHO) classification: Grade 0: No change; grade I: Soreness / erythema; grade II: Erythema and ulcers, patient can eat solids; grade III: Ulcers, the patient requires liquid diet only; and grade IV: Food intake is not possible. ${ }^{14}$ Intraoral charting of teeth and their status was done. The decayed, missing, and filled teeth per subject (DMF) for primary and for permanent dentition were recorded as per the WHO criteria. ${ }^{15}$ Oral hygiene status was evaluated as per Simplified Oral Hygiene Index given by Greene and Vermillion. ${ }^{16}$

Both extraoral and intraoral clinical examinations were done. Any enlargement or tenderness in the submental, submandibular, anterior and posterior cervical, and pre- and postauricular lymph nodes was recorded. The temporomandibular joints (TMJs) were palpated for any signs of pain or dysfunction. Intraorally, record of caries, gross tooth mobility, and tooth/jaw pain was made. Buccal and sulcular mucosa, the tongue, the floor of the mouth, the hard and soft palate, the fauces, and free and attached gingiva were examined systematically for evidence of any abnormality.

All the clinical data based on the examination of the patient were collected from the patients without distressing them and their attendants. Only the parents, attendants of the patients who wilfully provided consent for the examination and utilization of prevalent personal medical data from the hospital for the research work were included in the study and their written informed consent was recorded prior to the examination. No patient was subjected to any investigation for the study purposes.

\section{RESULTS}

Thirty-four males and 28 females, between the age of 2 and 14 years, were included in the study group. Their mean age was $7.42 \pm 3.619$ years. The various types of malignancy and the number of subjects suffering from each type are shown in Table 1 and Graph 1.

Table 1: Types of malignancy in the pediatric cancer patients

\begin{tabular}{lll}
\hline Type of malignancy & Number & Percent \\
\hline Hodgkin's lymphoma (HL) & 5 & 8.1 \\
Acute lymphoblastic leukemia & 22 & 35.5 \\
Osteosarcoma & 4 & 6.5 \\
Stem cell glioma & 1 & 1.6 \\
Glioblastoma & 2 & 3.2 \\
Acute myeloblastic leukemia & 4 & 6.5 \\
Astrocytoma & 2 & 3.2 \\
Non-Hodgkin's lymphoma (NHL) & 8 & 12.9 \\
Ca lung & 1 & 1.6 \\
Rhabdomyosarcoma & 3 & 4.8 \\
Neuroblastoma & 3 & 4.8 \\
Ewing's sarcoma & 4 & 6.5 \\
Anaplastic astrocytoma & 1 & 1.6 \\
Ca in situ & 1 & 1.6 \\
Hepatoblastoma & 1 & 1.6 \\
\hline Total & 62 & 100.0 \\
\hline
\end{tabular}




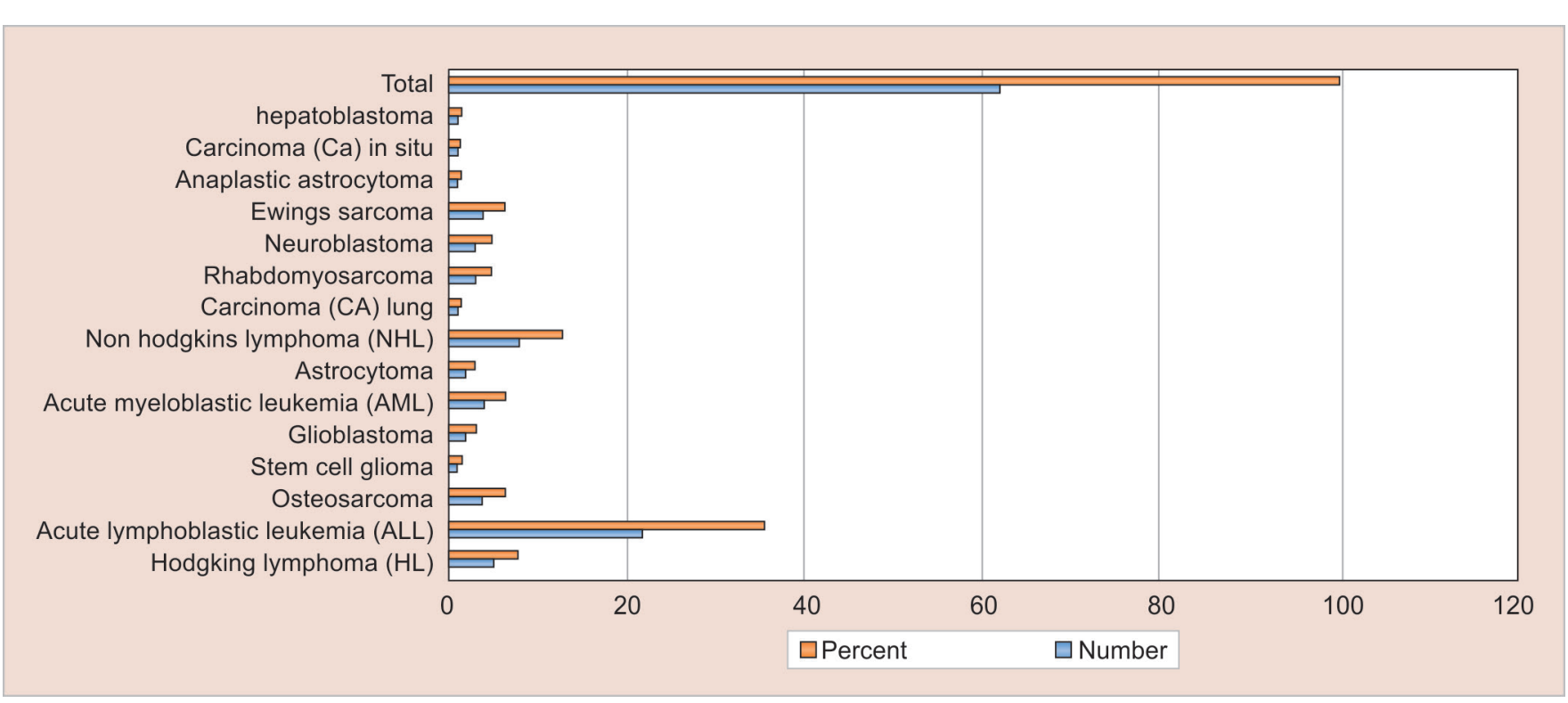

Graph 1: Distribution of malignancy in pediatric cancer patients

The most commonly encountered malignancy was ALL (35.5\%), followed by non-Hodgkin's lymphoma (12.9\%), then Hodgkin's lymphoma (8.1\%), and Ewing's sarcoma $(6.5 \%)$. However, the other types like neuroblastoma, hepatoblastoma, carcinoma lung were not frequent (Table 1). Various oral complications presented themselves coexisting with other complications in the subjects receiving chemotherapy (Table 2 ). On examination, oral mucosal ulcers and mucositis were the most frequently seen oral problems to occur during chemotherapy (Table 3). Out of 62 total subjects, 36 subjects suffered from varying degrees of oral mucositis. Clinically, symptoms

Table 2: Prevalence of oral and associated complications

\begin{tabular}{lll}
\hline Problem & No. of patients & Percent \\
\hline Head and neck lymphadenopathy & 7 & 11.2 \\
Lip cracking & 8 & 12.9 \\
Oral mucosal ulcers & 31 & 50 \\
Herpes simplex infection & 6 & 9.7 \\
Temporomandibular joint pain & 4 & 6.5 \\
Oral mucosal petechiae & 14 & 22.6 \\
Ecchymosis & 3 & 4.8 \\
Mucositis & 36 & 58.1 \\
Oral pain & 27 & 43.5 \\
Gingivitis & 24 & 38.7 \\
Candidiasis & 10 & 16.1 \\
Xerostomia & 22 & 35.5 \\
\hline
\end{tabular}

Table 3: Scoring of oral mucositis

\begin{tabular}{lll}
\hline & Oral mucositis & \\
\hline Subcategories & $n$ & $\%$ \\
\hline Grade I & 11 & 30.5 \\
Grade II & 14 & 38.8 \\
Grade III & 8 & 22.2 \\
Grade IV & 3 & 8.3 \\
\hline
\end{tabular}

observed varied from mild inflammation and redness of the mucosa to ulceration, with severe pain and bleeding (Fig. 1). Around $38.8 \%$ of patients had grade II mucositis while $8.3 \%$ of subjects had grade IV mucositis, which can lead to difficulty in oral food intake, severe pain, and at times necessitates the use of supplemental parenteral nutrition (Table 3 and Fig. 2). Ulcers were observed in 31 children and tended to appear 5 to 10 days after the start of chemotherapy. Secondary infection by Candida was seen in about five cases of mucosal ulceration in our study.

A significant proportion of subjects suffered complications related to bacterial and viral infections. Acute pseudomembranous candidiasis occurred in 10 patients, while 6 patients developed herpes simplex virus (HSV) infections. Four cases of HSV infection were seen in patients with ALL. One patient of Hodgkin's lymphoma and one of osteosarcoma suffered from HSV infection. Four subjects

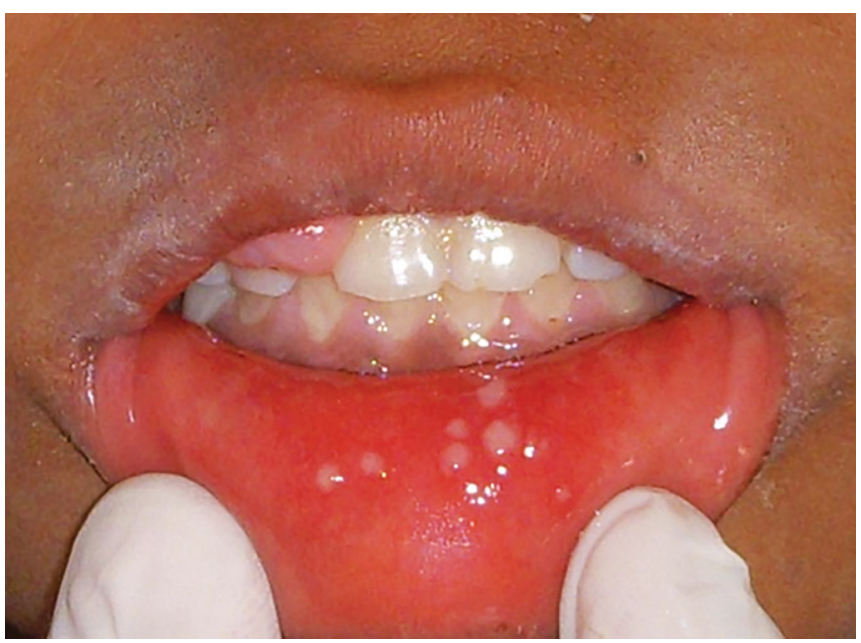

Fig. 1: Mucositis of lips with ulcers 


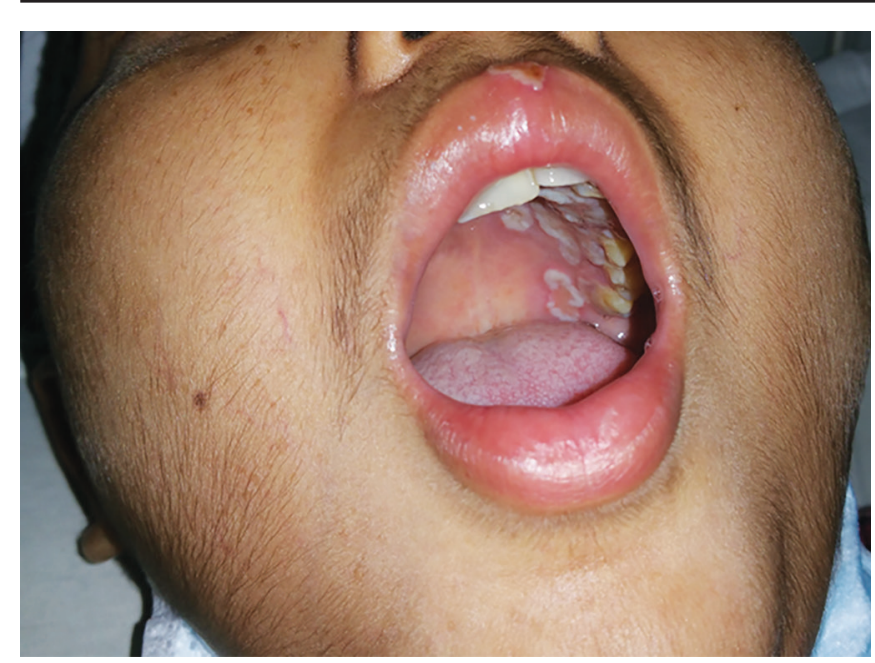

Fig. 2: Ulcers on palate and lips

had localized suppurative dental infection suggestive of bacterial cause. Thrombocytopenia presented as oral petechiae, especially on soft palate and buccal mucosa. Ecchymosis was observed in about three patients where PC was below $20,000 \mathrm{~mm}^{3}$.

\section{DISCUSSION}

Antineoplastic therapy in the form of radiotherapy and/ or chemotherapy can lead to an array of oral side effects which appear more frequently in the younger population. ${ }^{17,18}$ Systemic spread of pathogenic microorganisms can occur from the oral cavity through breach in the oral mucosal integrity induced by cytotoxic treatment that targets rapidly dividing cells. ${ }^{19}$ Any odontogenic infection can act as foci of sepsis which can cause serious life-threatening condition during periods of immune suppression. ${ }^{20}$ Marques and Walker ${ }^{21}$ have reported a case of severe facial cellulitis and secondary airway complication in the immunocompromised child due to a mobile exfoliating deciduous teeth.

Mucositis, aphthous ulcerations, secondary fungal or candida infections, xerostomia, oral pain, poor oral hygiene, gingival bleeding, and sialadenitis are some of the chemotherapy-induced oral complications manifesting in varying forms and degrees of severity. Such changes in the oral cavity can be painful, cause discomfort due to difficulty in eating and drinking, hence, interfering with and reducing treatment compliance. Necessary oral care at various stage of cancer treatment can prevent or reduce the incidence and severity of these oral complications. ${ }^{22}$ Late complications of the oncology treatment may include altered dental development and craniofacial changes that can cause severe cosmetic and functional sequelae requiring surgical and orthodontic interventions in the future. ${ }^{23}$
Most commonly seen nonhematologic complication of cytotoxic chemotherapy is oral mucositis. ${ }^{22}$ In the present study, $58 \%$ of the total subjects suffered from varying degrees of oral mucositis. Guggenheimer et $\mathrm{al}^{24}$ and Janković et $\mathrm{al}^{25}$ reported similar results in their studies, with 52 and $55 \%$ respectively, of the cancer patients suffering from oral mucositis. An increased prevalence (69\%) in pediatric cancer patients was observed in study by Wahlin and Matsson. ${ }^{26}$ This inflammatory condition generally begins 3 to 10 days after chemotherapy and can persist for 3 weeks. It has been shown to peak at around 7 to 14 days, at which time it slowly resolves unless complicated by infection. ${ }^{27,28}$ Mucositis can be all encompassing clinically presenting as mild inflammation to severe ulcerations, pain, bleeding, dysphagia, inability to eat/chew, drink, and talk, malnutrition, fatigue, and risk of both local opportunistic and systemic infection. ${ }^{29}$ Compromised oral intake and nutritional status accompanied with ulcerative pain can result in weight loss as well. ${ }^{29-31}$ In the present study, oral mucositis was clinically classified using WHO criteria (Table 3$).{ }^{14}$ Out of 26 subjects who were diagnosed with hematological malignancies, 16 had mucositis (61\%) as compared with $57 \%$ in subjects who had solid tumors, which is consistent with the findings demonstrated by Sonis et $\mathrm{a}^{31}$ that children with hematologic malignancies experience mucositis more frequently than those with solid tumors. Also certain chemotherapeutic drugs, such as doxorubicin, bleomycin, fluorouracil, and methotrexate and poor oral hygiene are associated with increased incidence of oral mucositis. ${ }^{30,32}$ The Multinational Association of Supportive Care in Cancer/International Society of Oral Oncology has published guidelines for the treatment of mucositis. The primary treatment of mucositis is palliative therapy, which includes home oral hygiene, pain control through use of analgesics or topical anesthesia, nonmedicated oral rinses (e.g., $0.9 \%$ saline or sodium bicarbonate mouth rinses 4-6 times/day), and symptomatic treatment of dry mouth. ${ }^{33}$ Recently, Palifermin, a keratinocyte growth factor- 1 in patients undergoing high-dose chemotherapy, is being considered. ${ }^{34}$

Oral pain was the next frequent oral manifestation in cancer patients undergoing antineoplastic therapy; $43.5 \%$ of total subjects had pain in the oral cavity accompanied with mucosal ulcerations and mucosal inflammation in most of the cases. Twenty-two subjects out of 27 who complained of oral pain in our study were receiving vincristine as part of their treatment. McCarthy and Skillings ${ }^{35}$ reported similar findings in their study and stated that oral pain in the absence of a dental/periodontal infection can also be a neurotoxic side effect of vincristine and vinblastine. They observed that a detailed clinical and radiographic study is further required to distinguish 
such pain from pulpal pain. ${ }^{17}$ In a study done by Miser et $\mathrm{al}^{36}$ in 1987 on 139 children and young adults, the evaluation of treatment-related pain revealed the most common cause to be mucositis, followed by postoperative pain and neuropathic pain due to vincristine. ${ }^{17}$

Immune prematurity, weakened host defense mechanisms can increase the susceptibility of an already immunocompromised patient to infections commonly bacterial and fungal in origin. Oral fungal infections particularly Candida are seen in patients with prolonged, severe neutropenic episodes leading to systemic complications, such as fungal esophagitis or even septicemia. Broad spectrum antibiotics, steroids, or any preexisting fungal infection further contribute to the risk. ${ }^{37,38}$ Herpes simplex virus is a common pathogen in cancer patients, which can aggravate oral mucositis. ${ }^{39}$ In most cases, reactivation of latent virus can lead to infections from HSV, varicella-zoster virus, and Epstein-Barr virus, while cytomegalovirus infection can result from the reactivation of a latent virus or from a recently acquired virus. ${ }^{17}$ Bacterial orodental infections usually caused by Gram-negative organisms are seen. Reduced inflammatory response in periods of myelosuppression can mask the symptoms, further causing difficulty in diagnosis. Compromised host defense, mucosal inflammation, or disruption can lead to serious infections by Streptococcus viridans, which is a part of normal oral microflora. ${ }^{37}$ Consequently, it is important that oral hygiene protocols should be planned and followed to reduce the level of microbial colonization of the dentition and periodontium particularly during the period of bone marrow suppression. In the present study, bacterial infections were seen among four subjects. Close monitoring of the oral cavity allows for timely diagnosis and treatment of fungal, viral, and bacterial infections. Microbial surveillance of all suspicious lesions should be performed and prophylactic medications should be initiated until more specific therapy can be (g chemo) prescribed. ${ }^{17,33}$

The American Academy of Pediatric Dentistry recognizes that the pediatric dental professional plays an important role in the diagnosis, prevention, stabilization, and treatment of oral and dental problems in pediatric oncology patients as a part of the multidisciplinary team approach. ${ }^{33}$ Emphasis is given to the role of the dental care team which should primarily focus on early diagnosis and preventive treatment so as to reduce the risk of infection and minimize the adverse effects of anticancer therapies. A detailed medical history of each cancer patient including information about the nature of disease, treatment planned, any episode of relapse, or complications should be taken in collaboration with the oncology team. The current hematological status, systemic review, or existing/potential source of any oral/systemic infection should also be considered. Pretreatment dental evaluation aims at recognizing and removal of any active and potential sources of infection in the oral cavity, possible sources of local irritation, such as a sharp tooth cusp or a rough area on a restoration, and oral cancer screening. ${ }^{33,40}$

\section{CONCLUSION}

Antineoplastic therapy can have adverse effects on the oral health of a cancer patient, which in turn can profoundly affect the general well-being of the patient. Oral mucositis, xerostomia, and opportunistic infections are more common in pediatric patients. Early involvement of a pediatric dentist can ensure effective oral care parallel to the ongoing oncological treatment, thus making a recognizable difference in the treatment success and palliative care. Professionally planned oral hygiene protocols, regular follow-ups, oral microbial surveillance, and palliative care to reduce oral discomfort caused due to antineoplastic therapy can help patients and their parents in combating the distressing yet unavoidable side effects of antineoplastic therapy. Further studies need to be conducted to evaluate on larger population to establish a correlation between various chemotherapeutic agents and oral or perioral complications and pain.

\section{CLINICAL SIGNIFICANCE}

Pediatric dental professionals are expected and play a key role in the multidisciplinary management of the pediatric cancer patients. The article aims to sensitize the pediatric dental professionals toward the management needs by highlighting the commonly encountered oral complications of such patients, which may vary according to the various chemotherapeutic agents used.

\section{ACKNOWLEDGMENTS}

The authors acknowledge the primary efforts and contributions of late Dr Ena Tiwari, Former Sr. Lecturer at the Department of Pedodontics and Preventive Dentistry. Inderprastha Dental College \& Hospital and would like to dedicate this article in her remembrance and fond memory.

\section{REFERENCES}

1. Parkin D, Pisani P, Ferlay J. Global cancer statistics. CA Cancer J Clin 1999 Jan-Feb;49(1):33-64.

2. Schochat SJ, Fremgen AM, Murphy SB, Hutchison C, Donaldson SS, Haase GM, Provisor AJ, Clive-Bumpus RE, Winchester DP. Childhood cancer: patterns of protocol participation in a national survey. CACancer J Clin 2001 Mar-Apr;51(2): 119-130.

3. Arora RS, Eden T, Kapoor G. Epidemiology of childhood cancer in India. Indian J Cancer 2009 Oct-Dec;46(4):264-273. 
4. Yaris N, Mandiracioglu A, Buyukpamukcu M. Childhood cancer in developing countries. Pediatr Hematol Oncol 2004 Apr-May;21(3):237-253.

5. Stiller, C. Childhood cancer in Britain: incidence, survival, mortality. 1st ed. Oxford: Oxford University Press; 2007.

6. Carl, W.; Sako, K. Cancer and the oral cavity. Chicago (IL): Quintessence Publishing Co; 1986.

7. Ried Haziest H, Jaffe N. Late effects of cancer treatment in children. Pediatr Dent 1995;17(4):273-284.

8. Carl W. Oral manifestation of systemic chemotherapy and their management. Semin Surg Oncol 1986;2(4):187-199.

9. Peterson DE, Sonis ST. Oral complications of cancer chemotherapy: present status and future studies. Cancer Treat Rep 1982 Jun;66(6):1251-1256.

10. Stafford R, Sonis S, Lockhart P, Sonis A. Oral pathoses as diagnostic indicators in leukemia. Oral Surg Oral Med Oral Pathol 1980 Aug;50(2):134-139.

11. Lowe O. Oral concerns for the pediatric cancer patients. J Pedod 1986 Fall;11(1):35-46.

12. Epstein JB, Stevenson-Moore P. Periodontal disease and periodontal management in patient with cancer. Oral Oncol 2001 Dec;37(8):613-619.

13. Guggenheimer J, Moore PA. Xerostomia: etiology, recognition and treatment. J Am Dent Assoc 2003 Jan;134(1):61-69.

14. Parulekar W, Mackenzie R, Bjarnason G, Jordan RCK. Scoring oral mucositis. Oral Oncol 1998 Jan;34(1):63-71.

15. World Health Organisation. Handbook for oral health surveys: basic methods. 4th ed. Geneva: WHO Offset Publication; 1997. p. $40-51$.

16. Greene JC, Vermillion JR. The simplified oral hygiene index. J Am Dent Assoc 1964 Jan;68(1):7-13.

17. López BC, Esteve CG, Perez MGS. Dental treatment considerations in the chemotherapy patient. J Clin Exp Dent 2011;3(1):e31-e42.

18. Bagattoni S, D'Alessandro G, Prete A, Piana G, Pession A. Oral health and dental late adverse effects in children in remission from malignant disease: a pilot case-control study in Italian children. Eur J Paediatr Dent 2014 Mar;15(1):45-50.

19. Azher U, Shiggaon N. Oral health status of children with acute lymphoblastic leukemia undergoing chemotherapy. Indian J Dent Res 2013 Jul-Aug;24(4):523.

20. Smith, MA.; Ries, LAG. Childhood cancer: incidence, survival, and mortality. In: Pizzo PA, Poplack DG, editors. Principles and practice of pediatric oncology. 4th ed. Philadelphia (PA): Lippincott Williams \& Wilkins; 2002. p. 1-12.

21. Marques AP, Walker PO. Intraoral etiology of a life-threatening infection in an immunocompromised patient: report of case. ASDC J Dent Child 1991 Nov-Dec;58(6):492-495.

22. El-Housseiny AA, Saleh SM, El-Masry AA, Allam AA. Assessment of oral complications in children receiving chemotherapy. J Clin Pediatr Dent 2007 Summer;31(4): 267-273.

23. Carrillo C, Vizeu H, Soares-Júnior LA, Fava M, Filho VO. Dental approach in the pediatric oncology patient: characteristics of the population treated at the dentistry unit in a pediatric oncology Brazilian teaching hospital. Clinics 2010 Jun;65(6):569-573.
24. Guggenheimer J, Verbin RS, Appel BN, Schmutz J. Clinicopathologic effects of cancer chemotherapeutic agents on human buccal mucosa. Oral Surg Oral Med Oral Pathol 1977 Jul;44(1):58-63.

25. Janković L, Jelić S, Filipović-Ljesković I, Ristović Z. Salivary Immunoglobulins in cancer patients with chemotherapyrelated oral mucosa damage. Eur J Cancer B Oral Oncol 1995 May;31B(3):160-165.

26. Wahlin $\mathrm{YB}$, Matsson L. Oral mucosal lesions in patients with acute leukemia and related disorders during cytotoxic therapy. Scand J Dent Res 1988 Apr;96(2):128-136.

27. Medina, PJ.; Shord, SS. Cancer treatment and chemotherapy. In: Talbert RL, DiPiro JT, Matzke GR, editors. Pharmacotherapy: a pathophysiologic approach. 8th ed. New York: McGraw-Hill; 2011.

28. Cheng KK, Molassiotis A, Chang AM, Wai WC, Cheung SS. Evaluation of an oral care protocol intervention in the prevention of chemotherapy induced oral mucositis in paediatric cancer patients. Eur J Cancer 2001 Nov;37(16): 2056-2063.

29. Stone R, Fliedner MC, Smiet AC. Management of oral mucositis in patients with cancer. Eur J Oncol Nurs 2005 Sep;9(Suppl 1): S24-S32.

30. Cheng KK, Chang AM. Palliation of oral mucositis symptoms in pediatric patients treated with cancer chemotherapy. Cancer Nurs 2003 Dec;26(6):476-484.

31. Sonis ST. Mucositis as a biological process: a new hypothesis for the development of chemotherapy-induced stomatotoxicity. Oral Oncol 1998 Jan;34(1):39-43.

32. Niehaus CS, Meiller TF, Peterson DE, Overholser CD. Oral complications in children during cancer therapy. Cancer Nurs 1987 Feb;10(1):15-20.

33. American Academy of Pediatric Dentistry. Guideline on dental management of pediatric patients receiving chemotherapy, hematopoietic cell transplantation, and / or radiation therapy. Am Acad Pediatr Dent 2013 Sep-Oct;35(5):E185-E193.

34. Stiff PJ, Emmanouilides C, Bensinger WI, Gentile T, Blazar B, Shea TC, Lu J, Isitt J, Cesano A, Spielberger R. Palifermin reduces patient-reported mouth and throat soreness and improves patient functioning in the hematopoietic stem-cell transplantation setting. J Clin Oncol 2006 Nov;24(33):5186-5193.

35. McCarthy GM, Skillings JR. A prospective cohort study of the orofacial effects of vincristine neurotoxicity. J Oral Pathol Med 1991 Aug;20(7):345-349.

36. Miser AW, Dothage JA, Wesley M, Miser JS. The prevalence of pain in a pediatric and young adult cancer population. Pain 1987 Apr;29(1):73-83.

37. Vento S, Cainelli F. Infections in patients with cancer undergoing chemotherapy: aetiology, prevention, and treatment. Lancet Oncol 2003 Oct;4(10):595-604.

38. Allen UD. Management of infections in the immunocompromised child: general principles. Lympho Sign J 2016 Aug;3(3):87-98.

39. Fayle SA, Curzon MEJ. Oral complications in pediatric oncology patients. Pediatr Dent 1991 Sep-Oct;13(5):289-295.

40. da Fonseca MA. Dental care of the pediatric cancer patient. Pediatr Dent 2004 Jan-Feb;26(1):53-57. 